Historic, Archive Document

Do not assume content reflects current scientific knowledge, policies, or practices. 

$\mathrm{pkt}$

Barr's Mammoth

Columbian White Mammoth

BEANS.

One quart will plant 100 feet in drills.

New Leopard Wax....

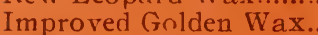

Flageolet Wax

Challenge Black Wax.

Wardwell's Early Kidney Wax..................................

Curries Rust Proof Wax.........................................

German Black Wax

Black Eyed Wax....

Davis White Wax......

Extra Early Valentine Green Pod..........................

Burpee's Stringless, Green Pod......

\section{LIMA BEANS.}

Extra Early Jersey Pole

King of the Garden Pole.

Burpee's Dwarf Bush Lima....

BEETS.

$1 \mathrm{oz}$ will plant 50 teet of drills, $6 \mathrm{lbs}$. an acre.

Detroit Dark Red .......................................... 5

Crosby's Imp. Extra Early Egyptian Blood Turnip 5

New Columbia Blood Turnip ............................ 5

Early Egrptian Blood Turnip................................. 5

Early Eclipse Blood Turnip ................................... 5

Early Edmund's Blood Turnip.............................. 5

New Dark Stinson Blood Turnip............................

Dewing's Improved Blood Turnip............................

Long Smooth Blood Red

\section{MANGEL WURTZEL BEETS.}

Golden Tankard.

Red Globe

Long Red

Vilmorin's Improved Sugar Beet.

CARROTS.

Early Scarlet Short Horn

Chanteney Half Long Scarlet ......................................

Danver's Half Long Orange......................................

Improved Long Orange.

CABBAGE.

Extra Early Jersey Wakefield Conical Shape........ 5

Early Charlston Wakefield Conical Shape.............. 5

Early Eclipse, Round Head ...................................

Burpee's All Head, Early Round Head......................

Faultless Large Early Round Head........................

Succession Large Flat Head ..................................

Sure Head ..........................................................

World Beater or Autumn King .............................

Select Prtmium Flat Dutch...................................

Danish Round Head or Holland..............................

Rock Red....................................................... 5

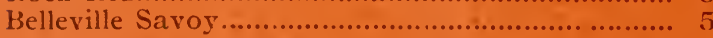

CAULIFLOWER. pkt

Extra Select Early Snowball ............................. 10

CUCUMBERS. pkt

$1 \mathrm{oz}$ to 50 hills. 2 lbs. to 1 acre.

Extra Early White Spine .................................... 5

Improved Long Early White Spine.......................... 5

Improved Long Green............................................ 5

Nichols' Medium

Westerfield's Chicago Pickling oz $\quad 1 / 4 \mathrm{lb}$

$8 \quad 20$

$8 \quad 20$

$\mathrm{lb}$

50

50

qt $\quad 4 \mathrm{qt}$

$\mathrm{pk}$

30

30

100

100

$\begin{array}{lll}30 & 1 & 00\end{array}$

$\begin{array}{lll}30 & 1 & 00\end{array}$

$30 \quad 100$

$30 \quad 100$

$\begin{array}{lll}30 & 1 & 00\end{array}$

$30 \mathrm{~J} \cdot 00$

$3 n$

1011

150

150

30

100

30

100

100

oz $\quad 1 / 41 b$

$\mathrm{lb}$

8

60

oz 
Perfected White Plume........................................ 5

Golden Selt Blanching.......................................... 5

Giant Pascal

Celeriac or Turnip Rooted ................................. 5

SWEET CORN.

$1 \mathrm{qt}$. to 200 hills. $8 \mathrm{qts}$. to 1 acre.

Peep ()' Day New Extra Early............................. 5 20

Extra Early New White Mexican........................... $5 \quad 20$

Extra Early White Cob Cory ............................... 5 5

Extra Early Fordhook........................................ 5

Early Minnesota ................................................ 5

Early Chicago Market.............................................. 5

Early Champion............................................... 5

Moore's Concord.................................................... 5

Country Gentleman....................................... 5

Late Mammoth Sugar....................................... 5

Stowell's Evergreen

ENDIVE. plst

Giant Fringed or Oyster....................................... 5

EGG PLANT.

Improved New York Purple Spineless................. 5

New Black Beauty ................................................ 5

KALE. pkt

Dwarf Green Curled......................................... 5

KOHL RABI or TURNIP ROOTED CABBAGE pkt

Early White Vienna...

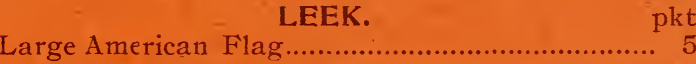

LFTTUCE. pkt

Early Black Seeded Curled Simpson..................... 5

Early Grand Rapids Curled.................................... 5

Early Ilenver Market Curled..................................

Early Hanson Hard Head...

MUSTARD.

Both White and Brown each

MUSK MELONS. pkt

Extra Early Hackensack.................................... 5

Extra Early Netted Gem ........................................... 5

Extra Early Emerald Gem................................ 5

Rocky Ford............................................................... 5

Paul Rose....................................................... 5

Osage

\section{WATER MELON. pkt}

Halbert Honey New ..................................... 10

Keckley's Sweet, Carefully Selected........................ 5

Keckley's Sweet Seed, grown by originator............ 10

Dark Icing ..................................................... 5

Ice Cream

Grey Monarch....................................................

McIver's Wonderful..................................................

Kolb's Gem .................................................... 5

Sweet Heart........................................................... 5

ONIONS.

Select Yellow Globe Danvers................................ 5

Large Red Wethersfield ........................................ 5

Prize Taker............................................................. 5

Extra Large Red Globe............................................. 5

Australian Brown (best keeper) ......................... 5

Southport White Globe............................................

White Portugal....................................................... 5

White Barletta (for pickling) ................................ 5

White Queen (for pickling) .... 
Bloomsdale

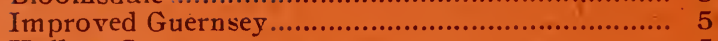

Hollow Crown.....

PUMPKINS. - pkt

Sugar Queen, best for pies.................................. 5

Large Connecticut............................................

Sweet Mountain........................................................ 5

Ruby King ....................................................... 5

Large Bell or Bull Nose........................................

Golden Dawn...................................................... 5

Red Cluster..................................................... 5

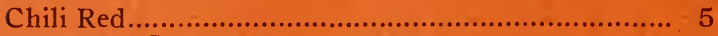

Long Red Cayenne............................................. 5

Celestial...................................................... 5

PEAS. pkt

$1 \mathrm{qt}$. to $75 \mathrm{ft}$. of drills.

List arranged in order as, to earliness.

Alaska, 2 feet high...

Maud S., 2 teet high....

Knott's Excelsior, 15 inches high .............................

American Wonder, 12 inches high.

Premium Gem, 11/2 feet high.

Dwarf Telephone.

Telephone.

Pride of the Market.

Champion of England.

RADISH. pkt

Early Scarlet Turnip.................................... 5

Early Scarlet Turnip, white tipped...................... 5

Early Rosy Gem, white tipped................................ 5

Early Round Dark Red ................................... 5

Early Ne Plus Ultra............................................... 5

Early French Breakfast, oval shape...................... 5

Long Brightest Scarlet, white tip........................ 5

Long Scarlet, short top................................... 5

Icicle.

White Lady Finger........................................ 5

White Strasbourg............................................... 5

Chartier

Rose China Winter...

Long Black Spanish Winter.................................... 5

SQUASH. $\mathrm{pkt}$

White Bush Súmmer Scolloped........................... 5

Yellow Bush Summer Scolloped. ............................ 5

Mammoth Yellow Summer Crookneck.................... 5

Early Fordhook, good for summer and wiuter.... 5

The Faxon..................................................... 5

Hubbard, the old standard....................................... 5

Leonard's New Warty Hubbard............................ 5

New Golden Hubbard........................................ 5

SALSIFY OR OYSTER PLANT. pkt

Sandwich Island Mammoth.............................. 5

SPINACH. pkt

Bloom sdale .....

pkt

Long Standing................................................. 5

New Victoria................................................................ 5

TURNIPS.

Extra Early White Milan.................................. 5

Extra Early Purple Top Milan............................. 5

Early Snowball ................................................. 5

Early White Flat Dutch..................................... 5

Red Top Strap Leaf............................................ 5

Purple Top White Globe...................................... 5

Bloomsdale Yellow Ruta Baga.......................... 5

$\begin{array}{rcr}\mathrm{oz} & 1 / 4 \mathrm{lb} & 1 \mathrm{~b} \\ 8 & 20 & 50 \\ 8 & 20 & 5() \\ 8 & 20 & 50 \\ \mathrm{oz} & 1 / 4 \mathrm{lb} & 1 \mathrm{~b} \\ 8 & 20 & 60 \\ 5 & 15 & 40\end{array}$

oz

50

25

25

25

25

25

25

25

25

qt $4 \mathrm{qt} \quad \mathrm{pk}$ 
Spark's Earliana (new)

New Quarter Century Dwarf Scarlet.........

Ponderosa

Dwarf Champion

Matchless

New Stone.

Favorite

Honor Bright.

Red Pear Shape....

Yellow Pear Shape.

5

25

SEED FIELD CORN.-Most popular varieties. Also CLovers, Buckwheat, Broov Corn. Sugar Cane, KafFir Corn, Brown's Inermis. Millet Rape Grass - eeds, at Market Prices. Please correspond.

POTATOES.

Extra Early Bovee.

Extra Select Early Ohio

Rural New Yorker No. 2

Carman's No. 3 .

$\left.\begin{array}{lll} & \mathrm{pk} & \text { Bush } \\ \text { Subject to } & 50 & 175 \\ \text { Market } & 50 & 175 \\ \text { Change }\end{array}\right\} \begin{array}{ll}17 \\ 50 & 175 \\ 50 & 175\end{array}$

PERFECTION LAWN GRASS.

$1 \mathrm{~b} \quad 5 \mathrm{lbs}$

bu of $14 \mathrm{lbs}$

Our mixture is the best possible..

$25 \quad 100$

250

\section{FLOWER SEEDS.}

Arranged Alphabetically. Price, exeept where noted, 5 cents per paper, 6 papers for 25 cents, postage paid.

Antirhinum or Snapdragon.

Amaranthus, Tricolor or Joseph's Coat.

Asters, mixed colors of the following kinds: Queen of the Market, Snowball, Poenie Flowered, Victoria, Gian Comet. Ostrich Feather, Daybreak, Semple's Late Branching.

Asters. Giant Comet and late branching in separate colors, at $10 \mathrm{c}$ per packet.

Browalia Elata, fine mixed.

Browalia Gigantea, 10c per packet.

Balloon Vine.

Belis or English Daisy,

Campanula or Canterbury Bells.

Canna, best kinds mixed.

Candytuft, mixed colors.

Celosia or Coxcomb.

Cobea Scandens, rapid climber.

Canary Bird Flower.

Cypress Vine.

Convolvulus or Morning Glory.

Carnation Marguerite.

Centaurea Cyanus or Corn Flower.

Caliopsis.

Cosmos, Early Flowering Dawn.

Cosmos, Mammoth Perfection.

Dianthus or Pinks.

Dolichos Lalab or Hyacinth Bean.

Datura Cornucopia or Horn of Plenty.

Escholtzia or California Poppy.

Forget Me-Not.

Gourds, fine mixed climbers.

Godetia.
Globe Amaranth.

Helichrysum (fine everlasting.)

Holly hock, best kinds mixed.

Humulisor variegated Japanese Hops.

Ipomea, New Imperial Japanese Morning Glory.

Larkspur. Dwarf Rocket, mixed.

Lobelia, both bushy and trailing kinds.

Marigold, best French mixed.

Marvel of Peru or Four O'Clocks.

Maurandia, fine climbing.

Mignonette, large sweet.

Mignonette, finest named kinds, $10 \mathrm{c}$ packet.

- Nasturtium, dwarf mixed, packet 5c, - ounce 15 cents.

Nasturtium, tall mixed, packet $5 c$, ounce 15 cents.

Pansy, choice mixed.

Pansy, extra choice mixed, packet $10 \mathrm{c}$.

Petunia, extra choice.

Phlox Drummondi, choicest kinds.

Portulacca, both single and double.

Poppy, finest mixed.

Ricinus or Caster Oil Bean.

Sweet Williams, double and single.

Stocks, German Ten Weeks.

Salpiglosis.

Smilax, most graceful climber.

Sweet Peas, finest kinds mixed, packet $5 \mathrm{c}$, ounce $10 \mathrm{c}$.

Thunbergia, free flowering climber.

Verbenas, choicest mixed.

Vinca Alba and Rosea.

Zinnia Elegans, best double. 


\section{FLOWERING PLANTS.}

For House and Oarden.

Of these I have a large assortment. The following and many others. Price except where uoted, small size 10 cents each, \$1.00 per dozen: Larger sizes 15 cents to 25 cents each, $\$ 150$ to $\$ 2.00$ per dozen.

Abutilon, Souv de Bonn. T. de Levet, Boile de Niege and Golden Bells. Alternanthera, 2 red leaved kinds, one yellow, 75 cents per dozen.

Ageritums, Stella Gurney and Princess Pauline.

Antirhinum or Snap Dragon, 8 hest colors.

Aloysia Citradora or Lemon Verbena.

Asparagus Plumosus, heautiful climber.

Asparagus Spengerii, beautiful climber.

Azalea Indica, glorious for winter and spring, $\$ 1.00$ to $\$ 2.00$ each.

Asters, all the best kinds and colors.

Begonia, best flowering kinds and for foliage.

Browalia Speciosa Major, best for the garden.

Cannas, 30 of the very best kinds. dry roots $\$ 1.00$ per dozen.

Cannas, plants from 4 inch pots, $\$ 1.50$ per dozen.

Carnations, best kinds, young plants 10 cents each, $\$ 1.00$ per dozen.

Carnations, larger plants in bloom, 25 cents to 35 cents each.

Chrysanthemums, best kinds young plants $10 \mathrm{c}$ each $\$ 1.00$ per dozen.

Chrysanthemums large plants in bloom, 25 cents to 50 cents each.

Coleus, 25 choice varieties of young plants, 75 cents per dozen.

Cobea Scandens, fine, very rapid climber.

Calla Lily, fine dry bulbs 10 to 20 cents each.

Calla Lily, plants in bloom, 30 to 75 cents each.

Caladium Esculentum or Elephant's Ear, dry bulbs 15 cents to 25 cents each.

Centaurea Gymnocarpa, white border plant.

Dahlias, over 50 best kinds, 15 cents each, $\$ 1.50$ per dozen.

Daisies (English) White, Pink and Red, 75 cents per dozen.

Fuchias, best single and double kinds.

Ferns, The true Boston Fern, 20 cents to $\$ 1.00$ each.

Ferns, The new Piersonii,. verv fine, 35 cents to $\$ 1.50$ each.

Ferns, the best kinds for fern dlshes.

Feverfew, Double White.

Geraniums, the best kinds from $2 \frac{1}{2}$ inch pots, 1 c each, $\$ 100$ per dozcn.

Geraniums, the best kinds from 3 inch pots, $121 / 2$ cents each, $\$ 1.25$ per dozen.

Geraniums, the best kinds from $31 / 2$ inch pots. 15 cents each, $\$ 1.50$ per dozen.

Geraniums, the best kinds from 4 inch pots. 20 cents each, $\$ 2$ no per dozen.

Gladiolas, best mixed 30 cents per dozen, separate colors, 50 cents per dozen.

Heliotrope, 3 best kinds.

Hydrangea. Thos. Hogg White and Otaksa Pink, 20 cents to $\$ 1.00$ each.

Hibiscus, best single and double, 15 cents to $\$ 1.00$ each.

Lantanas, 9 choice kinds.

Lillies, (Japanese) Auratum, Album and Roseum dry bulbs, 25 cents each.

Lillies, larger plants of above in bloom, 50 cents to 75 cents each.

Lillies, Harrisii and Longiflorem, the Easter lillies, dry bulbs 10 to 20c each.

Lillies, Harrisii and Longiflorem, plants in bloom, 35 cents to $\$ 1.00$ each.

Maugeurite or Paris Daisy, White and also Yellow.

Nerembergia, very tree flowering.

Petunia, 20 of the finest varieries.

Pelargoniums or Lady Washington Geraniums, 10 cents to 50 cents each.

Palms, all kinds, sizes and prices.

Plumbago Capensis, bnth the Blue and the White.

Primula or Chinese Primrose, all kinds.

Pansies, the very best strains from 30 to 40 cents per dozen.

Roses, monthly kinds the best kinds.

Roses. Crimson Rambler, also White, Yellow and Pink Ramblers, 35 cents to 50 cents each.

Roses, Harity Climbing. 6 varieties, strong 2 year old, 35 cents each.

Roses, best hardy perpetual kinds, strong 2 year old, $35 \mathrm{c}$ each, $\$ 3.50$ per doz.

Smilax, pretty climber, much used for decorations.

Salvias, Clara Bedman. Scarlet and Patens Blue.

Sweet Alyssum, double White, very sweet.

Torenia Fournieri, beautiful Pansy like fluwers, constant bloomers.

Tuberose, llouble Pearl, dry bulbs J cents each, 30 cents per dozen.

Tuberose, Double P'earl, plants in blonm 25 cents each.

Verbenas, best kinds, all colors, 3 for 25 cents, 75 cents per dozen.

Vinca or Running .Myrtle, 3 kinds, very fine for vases. 


\section{HARDY HERBACEOUS FLOWERING PLANTS.}

A One assortment, including the Rudbeckia or Golden Glow, Spirea Japonica, Hollyhocks. Phtox. Delphinium, Campanulas. Coreopis Lanceulata, Iris and Yucca Filamentose. Two sizes of plants; smaller, $15 \mathrm{c}$ each; larger size, 25c each. Peonies, a fine assortment, 25 to $50 \mathrm{cts}$ each.

\section{HARDY FLOWERING SHRUBS.}

Including Hydrangea Paniculata Grandifl sra or Garden Hydrangea, Spireas, Syringa, Dentzias. Wigelias. Snowbr 1s, Lilaes, Altheas, Almonds, Japan Quince, Acacia Rosea and others. $25 \mathrm{c}$ to $50 \mathrm{c}$ each.

\section{HARDY CLIMBING PLANTS.}

Ampelopsis Vietchii or Boston Ivy, Cinnamon Vine. Honeysuckle, Akebia Quinata, Begonia Radicans or Trumpet Creeper, Clematis, Paniculata Grandiflora. Strong plants of all above at 25 e each. Also Clematis Jackmanii at from $50 \mathrm{c}$ to $75 \mathrm{c}$, according to size.

\section{GRAPE VINES.}

\section{One dozen at price of 10 single vines.}

Concord ..........................1 yr. old $10 \mathrm{c} ; 2$ yrs. old. $15 \mathrm{c} ; 3$ yrs. old, $20 \mathrm{c}$. Agawam, Brighton, Diamond, Moore's Early, Niagara, Worden, Wilder and Campbell's Early...................................................2 yrs. old, 25c each

McPike's (new), hardy, vigorous, large and very sweet.....................35c each

\section{CURRANTS.}

Fay's Prolific, the best red..... 2 yrs. old, $10 \mathrm{c}$ each; $\$ 1.00$ per doz White Grape, the best white.........................2 yrs. old, $10 \mathrm{c}$ each; 1.00 per doz Black Champion, the best black................2 yrs. old, 10c each; 1.00 per doz

\section{GOOSEBERRY.}

Dowing, best American sort........................2 yrs. old, $10 \mathrm{c}$ each; $\$ 1.00$ per doz Industry, best English sort.......................2 yrs. old, 25e each; 2.50 per doz

\section{BLACKBERRIES.}

Ancient Britton, splendid, large and hardy.............per doz. 3nc; per $100 \$ 1.50$ Snyder, well known, hardy and prolific. per doz. 30c; per $100 \quad 1.50$

\section{RASPBERRIES.}

Red Cuthbert, well known, standard.....................per doz. 30c; per $100 \$ 1.50$ Red, Miller Red, large, fine flavor and hardy...........per doz. 30c; per $100 \quad 1.50$ Black Kansas, very large; prolific and hardy............per doz. 30c; per $100 \quad 1.50$ Black Liamond, first-class every way.....................per doz. 30c; per $100 \quad 1.50$

\section{FRUIT TREES.}

Apples, the best hardy kinds ...................................5 to $7 \mathrm{ft}$. high, $30 \mathrm{c}$ each Pear, Bartlett, Keiffer, Flemish Beauty and others......5 to $7 \mathrm{ft}$. high, $40 \mathrm{c}$ each Plum, Lombard, Ábundance and Miner....................5 to $7 \mathrm{ft}$. high, $4.0 \mathrm{c}$ each Cherry. Early Richmond. English Morella, Large

Montmorency and others....................... to $6 \mathrm{ft}$. high, $40 \mathrm{c}$ to $50 \mathrm{c}$ each

\section{EVERGREENS.}

Norwav Spruce..........................................2 to $4 \mathrm{ft}$. high, $25 \mathrm{c}$ to $75 \mathrm{c}$ each Arbor Vitae, American...................................2 to $3 \mathrm{ft}$. high, $30 \mathrm{c}$ to 5 () ceach ". ". Siberian........................................2 to $3 \mathrm{ft}$. high, 50c to $75 \mathrm{c} \mathrm{each}$ " " " Pyramidalis................................2 to $3 \mathrm{ft}$. high, $50 \mathrm{c}$ to $75 \mathrm{c}$ each Hemlock. $3 \mathrm{ft}$. high, $50 \mathrm{c}$ each.

\section{SHADE TREES.}

Larch, Catalpa, Mulberry, Mountain Ash........6 to $8 \mathrm{fr}$. high, 40c to $50 \mathrm{c}$ each Norway Maple, Kentucky Coffee Tree, Linden or Bass Wood, White Ash, Elm and Birch.........................6 to $8 \mathrm{ft}$. high, $50 \mathrm{c}$ to $75 \mathrm{c}$ each

\section{ASPARAGUS ROOTS.}

Barr's Mammoth and Columbian Mammoth, White......1 yr. old, $75 \mathrm{c}$ per 100 ". Mammoth and Columbian Mammoth, " 


\section{CUT FLOWER PRICE LIST.}

We Make a Specialty of this Department.

\section{Prices are always subject to changes, as to a large extent the weather governs prices.}

\section{Below Will be Found the Usual Prices in the Different Seasons.}

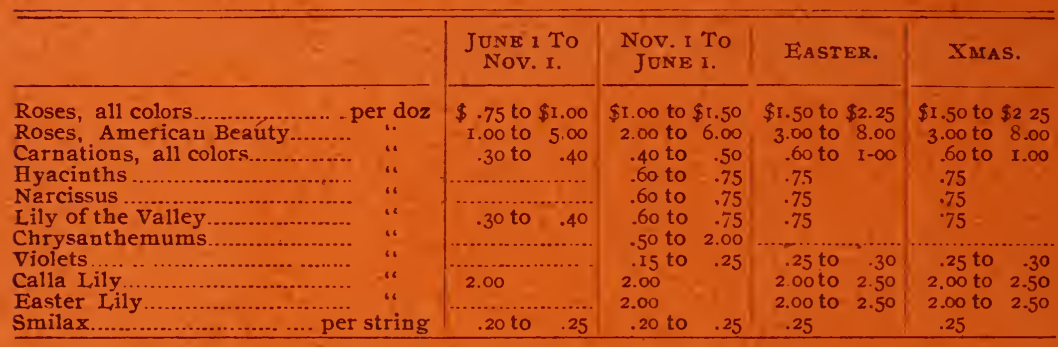

\section{Choice Floral Designs.}

Anchor, Flat. 12 inches, $\$ 2.00 ; 15$ inches, $\$ 3.00 ; 18$ inches, $\$ 5.00 ; 24$ inches, $\$ 6.00$.

Anchor, Standing.- 15 inches high, $\$ 4.00 ; 18$ inches, $\$ 5.00 ; 20$ inches, $\$ 6.00$ 24 inches, $\$ 7.00$.

Broken Column. 18 inches high, $\$ 4.00 ; 21$ inches, $\$ 5.00 ; 24$ inches, $\$ 7.00$ 3u inches, $\$ 10.00$.

Broken Wheel. 18 inches across, $\$ 6.00 ; 21$ in hes, $\$ 7.00 ; 24$ inches, $\$ 10.00$.

Crescent. 12 inches across, $\$ 2.00 ; 15$ inches, $\$ 3.00 ; 18$ inches, $\$ 4.00$.

Crosses, Flat. 12 inches. $\$ 2.00 ; 14$ inches, $\$ 2.50 ; 15$ inches, $\$ 3.00 ; 16$ inches, $\$ 4.00 ; 20$ inch's. $\$ 6.00 ; 24$ iuches. $\$ 7.50$.

Crosses, Standing. 18 inches high, $\$ 5.00 ; 20$ inches. $\$ 750 ; 24$ inches, $\$ 10.00$. 36 inches, $\$ 12.00$

Cross, Maltese. 10 inches, $\$ 2.50 ; 14$ inches, $\$ 3.50 ; 16$ inches, $\$ 4.00 ; 20$ inches, $\$ 5.00$.

Gates, Ajar. 20 inches high, $\$ 6.00 ; 22$ inches high, $\$ 7.50 ; 26$ inches high, $\$ 10.00$.

Harp. 15 inches high. $\$ 3.00 ; 18$ inches $\$ 4.00 ; 21$ inches, $\$ 5.00 ; 24$ inches, $\$ 6.00 ; 28$ inches, $\$ 750$.

Lyre. 15 inches high, $\$ 3.00,18$ inches, $\$ 4.00 ; 21$ inches, $\$ 5.00 ; 24$ inches, $\$ 7.50$.

Pillows. 10 inches long, $\$ 2.50 ; 12$ inches, $\$ 3.00 ; 15$ inches, $\$ 4.00 ; 16$ inches, $\$ 500 ; 18$ inches, $\$ 6.00 ; 21$ inches, $\$ 7.00 ; 24$ inches, $\$ 1000$. With Dove, $\$ 2.00$ extra.

Sickle. 12 inches long, $\$ 2.00 ; 15$ inches, $\$ 3.00 ; 18$ inches, $\$ 4.00 ; 24$ inches, $\$ 5.00$.

Sickle on Easel, with Sheaf of Wheat. 21 inches high, $\$+00 ; 2 t$ inches, $\$ 5.00$.

Shield. 12 inches across, $\$ 4.00 ; 16$ inches, $\$ 5.00 ; 18$ inches, $\$ 750$.

Stars. 10 inches, $\$ 2.00 ; 12$ inches, $\$ 3.00 ; 15$ inches, $\$ 4.00 ; 18$ inches, $\$ 5.00$.

Wreaths. 12 inches, $\$ 2.00 ; 14$ inches, $\$ 300 ; 16$ inches, $\$ 4.00 ; 18$ inches, $\$ 5.00 ; 20$ inches, $\$ 6.00 ; 24$ inches, $\$ 800$.

Flat Bouquets of Roses, Hyacinths, Chrysanthemums, Carnations, etc., for laving on caskets, tied with ribbon, $\$ 1.50, \$ 2.00, \$ 3.00, \$ 4.00$ and $\$ 5.00$ each. 